\title{
ZEITSCHRIFT FUR \\ PHYSIKALISCHE CHEMIE
}

BEGRONDET VON

WILH. OSTWALD UND J. H. VAN 'THOFF

HERAUSGEGEBEN IM AUFTRAGE

DER CHEMISCHEN UND DER PHYSIKALISCHEN GESELLSCHAFT IN DER DEUTSCHEN DEMOKRATISCHEN REPUBLIK VON

G. GEISELER, R. ROMPE,

F.SAUERWALD, W.SCHIRMER, K.SCHWABE,

E.STEGER, G. VOJTA

MIT 187 ABBILDUNGEN UND 67 TABELLEN

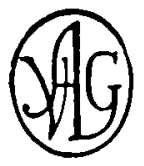

AKADEMISCHE VERLAGSGE SELLSCHAFT GEEST \& PORTIG K.-G. • LEIPZIG 